Xuemei Zhan, Zhong Hua Mu, Rajeev Kumar*, and Mohammad Shabaz

\title{
Research on speed sensor fusion of urban rail transit train speed ranging based on deep learning
}

https://doi.org/10.1515/nleng-2021-0028

Received Jun 14, 2021; accepted Aug 17, 2021.

\begin{abstract}
The speed sensor fusion of urban rail transit train speed ranging based on deep learning builds a userfriendly structure but it in-turn increases the risk of traffic that significantly challenges its safety and transportation efficacy. In order to improve the operation safety and transportation efficiency of urban rail transit trains, a train speed ranging system based on embedded multi-sensor information is proposed in this article. The status information of the train is acquired by the axle speed sensor and the Doppler radar speed sensor; however, the query transponder collects the status information of the train, and is used in the embedded system. Various other modules like adaptive correction, idling/sliding detection and compensation of speed transition/sliding are used in the proposed methodology to reduce the vehicle speed positioning errors due to factors such as wheel wear, idling, sliding, and environment. The results show that the running time of the train is 1000s, the output period of the axle speed sensor is $0.005 \mathrm{~s}$ and the accelerometer output period is 0.01 s.The output cycle of doppler radar is observed to be $0.1 \mathrm{~s}$, the output cycle of the transponder is $1 \mathrm{~s}$ and the fusion period of the main filter is observed as 1 s. The train speed ranging system of the embedded multi-sensor information fusion system proposed in this article can effectively improve the accuracy of the train speed positioning.
\end{abstract}

Keywords: information fusion, light speed sensor, the train, velocity ranging

Xuemei Zhan, Zhong Hua Mu, Zheng Zhou Railway Vocational \& Technical College, Henan Zhengzhou, 450000, China, E-mail: XuemeiZhan1@outlook.com, ZhonghuaMu2@outlook.com *Corresponding Author: Rajeev Kumar, Chitkara University Institute of Engineering and Technology, Chitkara University, Punjab, India, E-mail: rajeev.kumar@chitkara.edu.in

Mohammad Shabaz, Arba Minch University, Ethiopia,

E-mail: Mohammad.shabaz@amu.edu.et

\section{Introduction}

The urban rain transit system provides a user-friendly vehicle transportation support that tracks the passenger carrier and guides the passengers regarding urban railways [1]. The passengers highly rely on such systems and if any fault occurs, significant losses are to be faced by the people. Thus, railway safely is very critical for ensuring the safety of passengers and the society, however, with the modern development in the field of high-speed railways directly affect the safety measures. The research in the field of speed measurement and train position monitoring is the utmost requirement and provides an important research direction.

The on-board controller (VOBC) of urban rail transit train is responsible for completing the functions of on-board train automatic protection (ATP) and automatic train operation (ATO). The main function of on-board ATP is to control the train running interval according to the immediate speed and travel distance of the train. Itprevents the train from over-speeding and ensure the safety of the train running. The function of the on-board ATO is to control the comfortable, energy saving and efficient operation of the train based on the immediate speed and travel distance of the train. Therefore, the train speed and traveling distance information are the basic parameters to ensure the normal operation of VOBC, and its accuracy and reliability directly affect the safety and efficiency of the train operation [2].There is a need to develop a precise and reliable real time system that can provide the train position as well as speed estimation for safeguarding the passengers while guaranteeing the normal operation of the train [3-5].

The sensor-based methodology suits best for the measurement of speed and position [6, 7]. Figure 1 depicts different types of speed and position sensors.

Wheel and axle speed sensors are widely used in urban rail transit to measure the speed and distance of the train in real time. Wheel and axle speed sensor is economical and practical, and the technology is well established $[8,9]$. By measuring wheel speed, the train speed and distance can be obtained more accurately. However, once the speed measuring wheelset idles/coasting, there will be a large deviation between the wheel speed and 


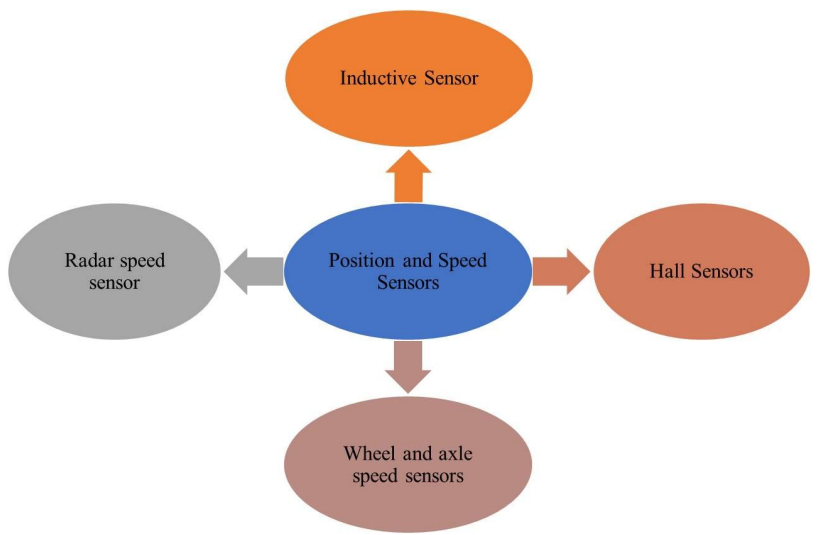

Figure 1: Different speed and position sensors

the actual running speed of the train, resulting in a significant increase in the measurement and ranging error of the wheel shaft speed sensor. The wheel shaft speed sensor itself cannot effectively solve the problem. In order to avoid idling/taxiing as much as possible, railway operating departments have taken certain adhesive control measures for the train operation, which can avoid serious idling/taxiing to a large extent, but a large number of weak idling/taxiing still exist. Thelow and high frequency idling/taxiing are caused by the train speed measurement and ranging error, and they constitute the essence of the train speed measurement and ranging method based on the wheel and axle speed sensor location error. This limitation of the existing methodology is solved by using the auxiliary positioning equipment (such as query/transponder, etc.) to provide point position information to the train and correct the cumulative ranging error of the wheel and axle sensors. However, still there are several disadvantages such as the failure to provide speed correction, high construction and maintenance costs, and the failure to support dynamic configuration changes of the line [10].

This article addresses all these concerns in the conventional speed and position measurement systems and significantly improves the autonomous positioning ability of the train. The major contributions of this article are:

- It introduces the fusion the doppler radar into the existing train speed measurement and ranging methodbased on the wheel and axle speed sensor, and uses the combination of the two to construct the speed measurement and ranging system.

- A train speed ranging system based on embedded multi-sensor information is proposed in this article that controls the status information of the train by employing axle speed sensor and the Doppler radar speed sensor.
- The query transponder collects the status information of the train, and is used in the embedded system for error reduction in the vehicle speed positioning due to factors such as wheel wear, idling, sliding, and environment.

- The calculation model of idling/taxiing detection and error correction is established to realize the effective detection and error correction of idling/taxiing.

- The purpose of improving the accuracy and reliability of train speed measurement and ranging is achieved using the proposed methodology [11].

The rest of this article is organized as: Section 2 presents the survey of literature in the field of position monitoring and speed estimation. Section 3 elaborates the research methods used in this article followed by the simulation analysis and results in Section 4. The conclusion of the article is presented in Section 5.

\section{Literature survey}

The measurement and ranging system of urban rail transit train requires real-time, continuous and stable measurement and ranging results as the vehicle control basis of the on-board automatic protection system. The speed ranging system can use a variety of combinations of speed sensors, including inertial navigation device (INS), tachometer, doppler radar, GPS, etc. Due to the disturbance of the train traction force and braking force output, the track friction and ground slope change constantly, the train speed and acceleration present a complex process of change; on the other hand, the sensor pulse acquisition process also has noise interference. In the velocity ranging processing module, it is necessary to analyze the characteristics of the output signal of the sensor and establish an appropriate filtering model to obtain smooth velocity ranging data. At the same time, the processing module should also integrate the working state and output data of each sensor to get the results of velocity measurement and ranging after fusion. At present, many scholars have conducted indepth studies on the speed measurement and ranging system of urban rail transit trains: Lin et al. proposed a speed and distance measurement algorithm based on the multisensor information fusion of wheel speed sensor and acceleration sensor. Because when the wheel speed sensor is accurate, when the wheel does not slide, the acceleration sensor is not affected by the wheel slip, so the algorithm uses these two types of sensors to judge whether the wheel is sliding and uses different information fusion 
methods. The wheel speed and train speed are calculated by the wheel state. Through field test, this algorithm can effectively detect whether the wheel is sliding and calculate the actual speed of the train, which has the practical application value [12]. Xiong et al. carried out the study on passenger flow in Beijing subway stations and lines, and compared the prediction results of deep learning method with several traditional linear models, including ARIMA, SARIMA and space-time autoregressive integrated moving average (STARIMA). The results show that LSTM NN and CNN can better capture the temporal or spatiotemporal characteristics of urban rail transit passenger flow, and obtain accurate short-term passenger prediction results. Deep learning methods also have strong data adaptability and robustness, and they are more suitable for predicting passenger flow at stations during peak periods and passenger flow on holiday lines [13]. Dai et al. used the inductive technology for the detection of alternating magnetic field and achieved the reliable and efficient train position [14]. This methodology employed the common mode interference of vehicle antenna but involves high engineering cost and has maintenance load as a drawback.

Liu et al. employed a comparative study for obtaining the information of different acceleration sensors as well as the radar sensors [15]. The method proposed by the authors measures the speed and position of medium to low speed trains with great efficiency. Harrer et al. proposed a signal detection methodology for providing a real time measurement and tracking [16]. This technique uses the combination of compensationand switching techniques for effective elimination of error and noise. The fusion method utilized in this work is not widely applicable and has confined scope. The measurement accuracy was improved by Yang et al. exploiting the doppler radar and automated correction phenomenon of double antennas [? ]. This integration is reliable and proves the error estimation and control in speed measurement practices. However, real time implementation is still not feasible due its nonapplicability in the dynamic working environment. Liu et al. proposed a significant method for correcting the speed measurement deviation using the radar velocity calculation [18]. This methodology involves inherent deviation correction method that is accompanied by the complex working conditions. Zhang et al. used the global satellite navigation system for the application of position measurement in trains [19]. They utilized the combination of zero speed correction and inertial navigation to be utilize for military application considering the information fusion development. Some of the research in this field relies on the utilization of global navigation along with transponder and pulse width coding phenomenon [20-22]. This tech- nique can be used for suspecting the positioning errors in the train transportation. The data fusion algorithm is combined with the GPS signaling by Zhang et al. in [23] and this combination reduces the errors during the train positioning. A verity of complex issues is being targeted by this methodology and a theoretical support is provided for real speed positioning. Ma et al. developed a system for China's railway industry by using the GPS modelling to avoid the probable risk factors in single speed positioning [24]. This methodology realizes the relative position errors and introduces the machine learning knowledge protocol for the evaluation of sensor states, thereby, improving the overall reliability of the system.

This literature review provides several shortcomings of the existing work like lack of dynamics, high engineering cost, workload for maintenance, complex working conditions, and many more. These issues are needed to be resolved by the current methodology in order to provide a reliable solution for train positioning, its speed measurement and ranging. This article suggests an embedded multi-sensor information for controlling the status information of the train.

\section{Research methods}

Various sensor combination schemes, vehicle speed measurement and ranging system, filtering and other processing of multi-sensor information fusion are used as the research methods in the proposed embedded multi-sensor information fusion system. All these methodologies are detailed in the following subsections.

\subsection{Sensor combination scheme}

\section{(1) Axle speed sensor}

Wheel and axle speed sensor can convert the wheel speed into electric pulse proportional to the train running speed. By collecting the pulse signal, the train's immediate speed and distance can be calculated. In this paper, the vehicle HS221G1A wheel shaft pulse velocimeter is used, and the velocimetry range is $0 \sim 20 \mathrm{kHz}$. The calculation formula of train speed and traveling distance is as follows: 


$$
\begin{gathered}
v=\frac{\Delta n \cdot \pi \cdot D}{N \cdot T} \\
\Delta S=\frac{\Delta n \cdot \pi \cdot D}{N}
\end{gathered}
$$

Type:

$v$ - The train speed $(\mathrm{m} / \mathrm{s})$;

$N-$ The number of pulses emitted by the sensor per wheel revolution;

$D$ - The wheel diameter (m);

$\Delta n$ - Pulse measurement value of this period;

$T$ - Speed cycle (s);

$\Delta S$ - Distance traveled by the train (m).

Because the wheel and axle speed sensor measures the wheel rotation speed, when the train is running normally, the wheel speed and the train running speed are basically the same; however, when the wheel idles/coasting, there will be a large deviation between the wheel speed and the actual running speed of the train, leading to a significant increase in the velocity measurement and ranging error of the wheel shaft speed sensor, and the ranging error will increase with the accumulation of train operation [25].

\section{(2) Radar speed sensor}

Doppler radar (doppler radar) speed measurement is based on the vehicle mounted on the bottom of the locomotive to send electromagnetic wave to the track surface and receive the reflected echo signal, based on the principle of doppler frequency shift effect by measuring the radar transmitted wave and reflected wave frequency difference (doppler frequency shift) can calculate the immediate speed of the train. The train traveling distance can be obtained by integrating the speed. DRS05A vehiclemounted radar is used in this paper, and the speed measurement range is $0.2 \sim 600 \mathrm{~km} / \mathrm{h}$. The velocity calculation formula is as follows:

$$
v=\frac{f_{r} \cdot \lambda}{2 \cos \theta}
$$

Type:

$f_{r}-$ Doppler shift $(\mathrm{Hz})$;

$\theta$ - The Angle between the radar transmitting wave and the orbital plane $\left(^{\circ}\right)$;

$\lambda-$ The transmitting wavelength of the radar $(\mathrm{m})$.

Since radar speed measurement does not depend on wheel rotation and is not affected by idling/sliding of wheelset, the error of speed measurement is mainly caused by longitudinal vibration of train and error of radar installation angle. Wheel-axle speed sensor and doppler radar have good complementarity. When the train runs at high speed, due to the obvious doppler effect, the radar has higher speed measurement accuracy, while when the train runs at high speed, the empty rotation/sliding of the wheelset is more frequent than when the train runs at low speed, because the accuracy of the wheel axle speed sensor is relatively low. When the train is running at low speed, the precision of the wheel shaft speed transducer is higher, while the precision of the radar is lower because of the inconspicuous doppler frequency shift effect. As the price, volume and accuracy of doppler velocity radar are reduced and improved, it has been applied in the field of velocity measurement and ranging of rail transit trains. Wheel - axle velocity transducer and radar speed transducer are a reasonable combination scheme of vehicle borne sensors.

\subsection{Vehicle speed measurement and ranging system}

\section{(1) System hardware}

A redundant configuration of two axle sensors and two radar sensors is used to improve system reliability. The hardware structure of the system is shown in Figure 2, in which the signal acquisition module realizes the synchronous acquisition and preprocessing of the information of each sensor. Because the wheel shaft and radar sensor belong to the high frequency continuous relative positioning, the low frequency discontinuous absolute positioning information is needed to assist. In this design, the ground transponder is used as a reference positioning system, one is to provide the initial position information for the train, the other is to use the accurate position information provided by the transponder to evaluate the measurement results of the vehicle speed ranging system.

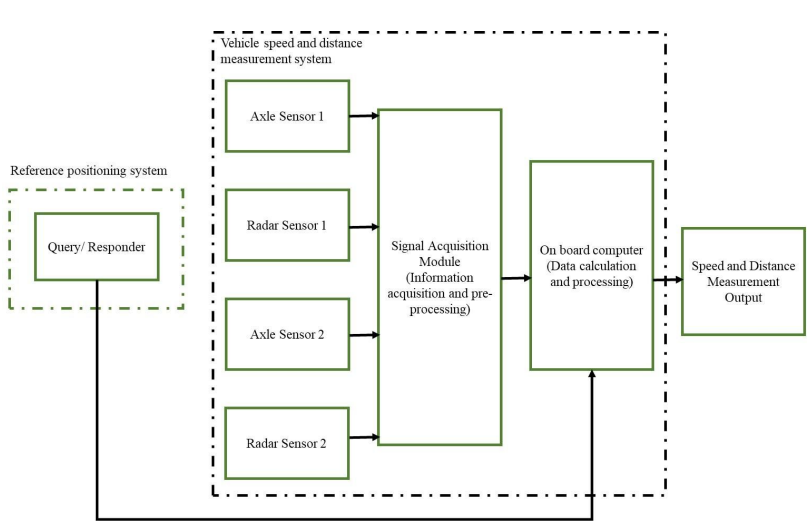

Figure 2: Hardware structure of velocity measurement and ranging system 


\section{(2) The system software}

The system software mainly calculates and processes the measured data of the speed sensor, realizes the algorithm model designed in this paper to complete the idling/taxiing detection and error correction, and at the same time receives the accurate position information provided by the ground transponder to complete the evaluation of algorithm performance. The overall structure of system software function modules is shown in Figure 3.

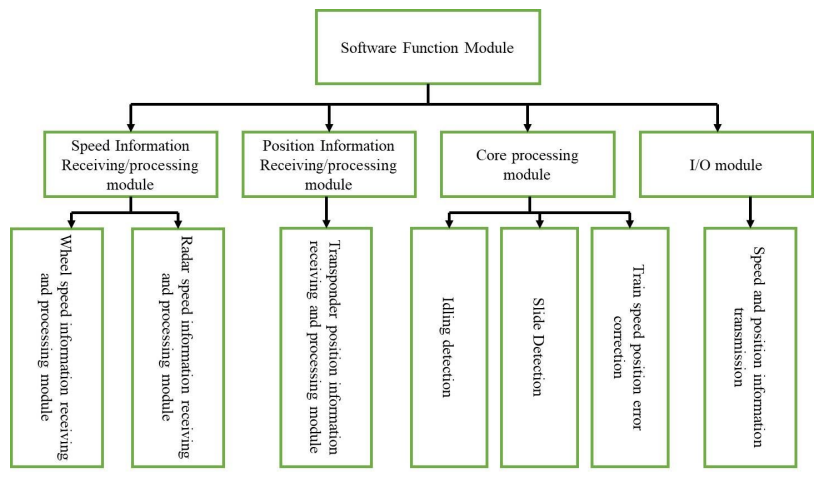

Figure 3: Software functional module structure

\subsection{Processing process of multi-sensor information fusion}

\section{(1) Multi-sensor combination}

The train speed measurement and positioning technology using a single sensor has its own disadvantages. The whole system cannot work properly due to occasional faults.The advantage of the multi-sensor information fusion technology is that it can provide more accurate and reliable information for the speed measurement and positioning system through redundancy, complementarity and combination of more kinds of information. Multi-sensor combination plays a very important role in train speed measurement and positioning. The phenomenon of idling and taxiing can be detected accurately by using the difference of acceleration measurement mechanism between the wheel and axle speed sensor and acceleration sensor. The speed measurement principle of the wheel shaft speed sensor and the doppler radar speed sensor are completely different, and their error sources are also very different. They come from different situations and are unrelated to each other. The wheel shaft speed sensor and the doppler radar speed sensor can effectively complement each other; the function of the ground query transponder sensor is to correct the discrete point of the train po- sition and the wheel diameter of the train without idling and taxiing through the absolute train position information sent by the ground. Among them, the train speed and position correction principle of multi-sensor information fusion is shown in Figure 4 [26].

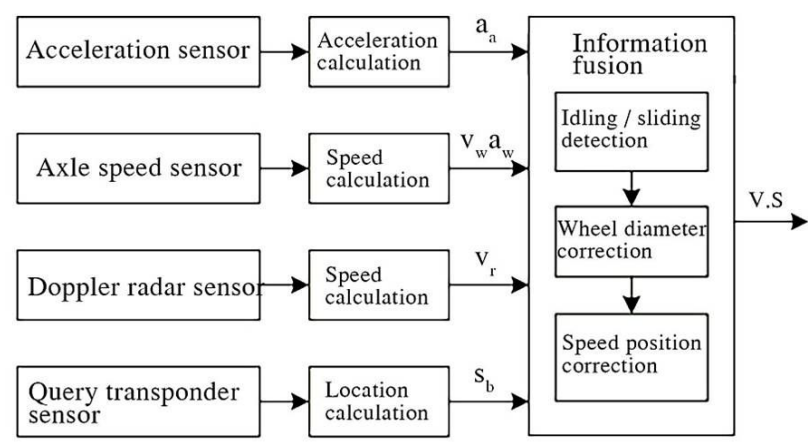

Figure 4: Principle of multi-sensor information fusion

(2) Idling/taxiing detection and wheel diameter correction

The acceleration detection of the wheel shaft speed sensor and the acceleration sensor are based on different measurement principles. The measurement equation of the wheel shaft speed sensor is shown in Eqs. (4) and (5), and the measurement of the acceleration sensor is shown in Eq. (6).

$$
v_{w}=\left(1+y_{w}\right) \cdot v_{w l}(k)+v_{s}(k)+N_{w}(k)
$$

$$
a_{a}(k)=a_{w l}(k)+\frac{\Delta v_{s}(k)}{T}
$$

$$
a_{a}(k)=a_{a l}(k) \cdot \cos \theta(k)+g \cdot \sin \varphi(k)+\varepsilon_{a}(k)+N_{a}(k)
$$

Where, $v_{w}(k)$ is the real speed measurement value of the axle speed sensor; $v_{w l}(k)$ is the true value of the axle speed sensor; $y_{w}$ is the wheel diameter error ratio; $v_{s}(k)$ is the error caused by idling slip; $N_{w}(k)$ is the noise in the measurement process; $N_{w}(k)$ can be ignored after differentiation and filtering; T is the adoption period; $a_{w}(k)$ is the real acceleration measurement value of the axle speed sensor; $a_{a}(k)$ is the real measured value of the acceleration sensor; $a_{a l}(k)$ is the real acceleration of the train; $\theta(k)$ and $\varphi(k)$ are the installation errors of the accelerometer; $g$ is the acceleration of gravity; $\varepsilon_{a}(k)$ is the random error in the measurement process; $N_{a}(k)$ Iis the measurement noise of acceleration.

The idling/sliding acceleration detection schematic diagram is shown in Figure 5. Where, solid lines of OA and 
DG represent the normal traction process of the train; Ka and $\mathrm{Dh}$ represent the normal braking process; the dashed line $\mathrm{AD}$ represents the idling and taxiing process of the train. ABCD represents the acceleration value measured when the wheel shaft speed sensor is idling; AFED stands for the acceleration measured by the axle speed sensor when gliding; $\mathrm{AD}$ represents the acceleration value measured by the acceleration sensor during idling/sliding; $\Delta t_{1} \sim \Delta t_{4}$ represents four possible forms of acceleration measurement periods [27].

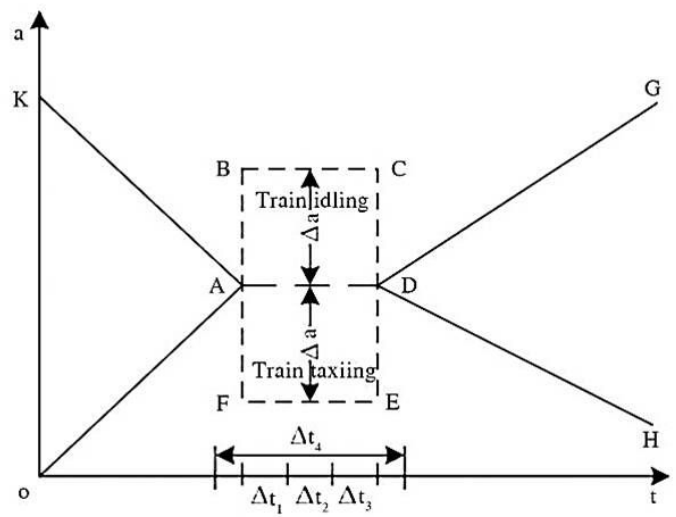

Figure 5: Schematic diagram of idling/gliding acceleration detection

When the train runs normally until time $\mathrm{A}$, the train will be in idling or taxiing operation state respectively because the traction force is greater than the adhesive force or the braking force is greater than the adhesive force. When the train is idling or taxiing, the train, as an inertial body, uses the wheel and axle speed sensor to measure acceleration $a_{w}$ no longer follows the principle of Eq. (5), while the acceleration A measured by the acceleration sensor is still valid, the idling/gliding phenomenon can be accurately identified by using the difference between the two values $\Delta a$.

$$
\Delta a=a_{a}(k)-a_{w}(k)
$$

$0\langle\omega\langle\Delta a$ refers to the idling phenomenon of train transmission; $\Delta_{a}\langle-\omega<0$ refers to the phenomenon of train sending and sliding. Where, $\omega$ is the upper and lower limit when idling/gliding occurs.

The train wheel diameter correction is to obtain the relative position information of the train on the track by using the query-transponder installed on the ground. When the train runs normally in two adjacent transponder regions, the distance between the beacons can be accurately obtained. Because of the safety coding characteristics of the wheel shaft speed sensor, the wheel diameter correction can be carried out. The correction principle is shown in Eq. (8):

$$
d=\frac{n \times s_{b-b}}{\pi \times N}
$$

Where, $s_{b-b}$ is the relative distance between adjacent transponders $(\mathrm{mm})$; $\mathrm{N}$ is the number of detection pulses between adjacent transponders.

\section{(2) Speed and position compensation}

The speed measured by the axle speed sensor is the rotation speed of the wheel itself. When the train sends idling or taxiing phenomenon, the train has both running speed and idling or sliding speed. The speed of the car body is no longer equal to the speed measured by the axle speed sensor. The speed measured by the radar speed sensor is only the speed of the vehicle body, and the measurement equation is shown in Eq. (9). However, there is a certain error relative to the wheel and axle speed sensor. The information of the two sensors can be fused to effectively compensate the speed and position.

$$
v_{r}(k)=\left(1+y_{r}\right) \cdot v_{r l}(k)+\varepsilon_{r}(k)+N_{r}(k)
$$

Where, $v_{r}(k)$ is the real measured speed of the train body; $y_{r}$ is the installation error; $v_{r l}(k)$ is the actual speed of the train body; $\mathrm{A} \varepsilon_{r}(k)$ is the random error in the measurement process; $N_{r}(k)$ is the measurement noise.

As shown in Figure 6, the broken line is the speed W2LOK measured by the axle speed sensor, means the train idling and taxiing; the dashed line represents the actual running speed of the train $\mathrm{v}$; the thick line represents the speed $v_{r}$ of the train measured by the doppler radar velocimeter.

In the speed measurement period $\Delta t$ when idling/taxiing occurs, the train has both idling/taxiing and moving. Therefore, the speed measurement value of the train wheel and axle sensor includes both the moving speed and the idling/taxiing speed of the train. The speed detected by doppler radar is always the speed of the train. According to the speed measurement principle of the vehicle-mounted speed sensor, it can be known that:

$$
\begin{gathered}
v_{w}=\frac{v_{k / h} \times \Delta t_{k / h}+v_{z} \times \Delta t_{z}}{\Delta t_{k / h}+\Delta t_{k / h}} \\
v_{r}=v+\delta
\end{gathered}
$$

Where, $v_{k / h}$ is the idling speed of the train; $t_{k / h}$ is the idling time of the train; $\Delta t_{z}$ is the travel time of the train; $\delta$ is the velocity measurement error of doppler radar sensor. The travel distance of the train is:

$$
S=\frac{\left(v_{0}+v_{w}\right) \times \Delta t}{2}
$$




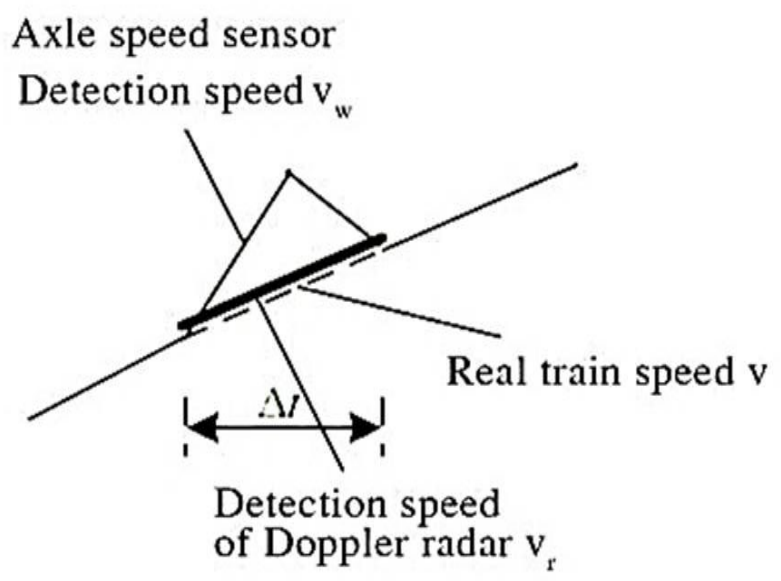

(a) Idle compensation

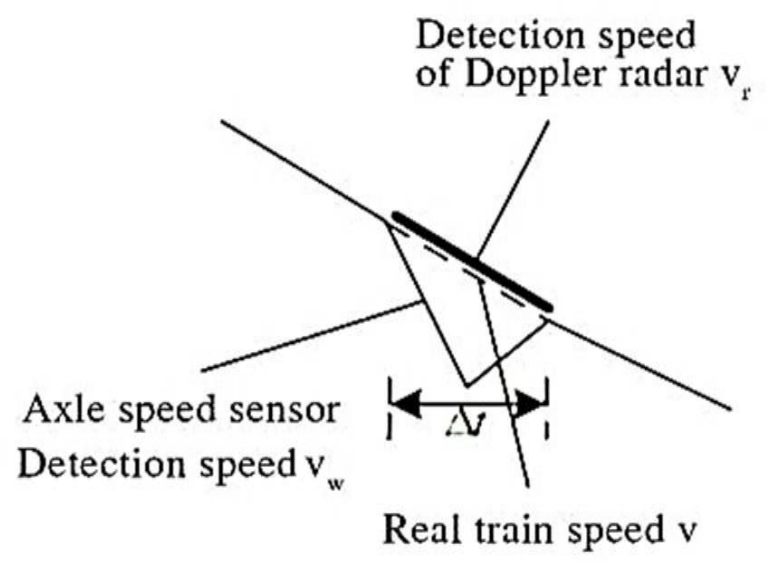

(b) Taxiing compensation

Figure 6: Schematic diagram of idling/taxiing compensation

Where, $v_{0}$ is the speed of the train at a moment.

Because the system detects the idling/skidding phenomenon of the train, the measured speed value $v_{w}$ of the wheel and axle speed sensor cannot represent the running speed of the train, so $\mathrm{S}$ is not the actual traveling distance of the train. The actual travel distance of the train should be revised to $S^{\prime}$ and the speed to $V^{\prime}$

$$
\begin{gathered}
S^{\prime}=\frac{\left(v_{0}+v_{r}\right) \times \Delta t}{2}=\frac{\left(v_{0}+v+\delta\right) \times \Delta t}{2} \\
v^{\prime}=\frac{v_{0}+v_{r}}{2}
\end{gathered}
$$

Assume that the train is traveling at a constant speed of $v_{0}$ or a constant speed of $v_{r}$ in time $\Delta t$, then, the distance traveled by the train in time $\Delta t$ is $v_{0} \times \Delta t$ or $v_{r} \times \Delta t$, then the range of error $\lambda$ of the train traveling distance is:

$$
\left[v_{0} \times \Delta t-\frac{\left(v_{2}+v+\delta\right) \times \Delta t}{2}, v_{r} \times \Delta t-\frac{\left(v_{0}+v+\delta\right) \times \Delta t}{2}\right]
$$

Namely:

$$
\lambda \in\left[\frac{\left(v_{0}-v_{r}\right) \times \Delta t}{2}, \frac{\left(v_{r}-v_{0}\right) \times \Delta t}{2}\right]
$$

If the train is idling/coasting only in the time of the speed measurement period $\Delta t$, then the train's traveling speed depends entirely on the doppler radar velocimeter. Therefore, the train's traveling error depends on the speed measurement accuracy of the doppler radar velocimeter. The maximum difference of train travel distance can be obtained as $(v+\delta) \times \Delta t-(v-\delta) \times \Delta t$. Therefore, the error range of the train travel distance is as follows:

$$
\lambda \in[0,2 \times \delta \times \Delta t]
$$

According to Eqs. (15) and (16), the smaller the speed measurement period $\Delta t$ and the speed measurement error $\delta$ of Doppler radar are, the smaller is the error of the train traveling distance.

\subsection{Filtering processing}

Current studies show that Kalman filtering algorithm is widely used in train speed measurement and positioning. In this study, federated Kalman algorithm is adopted for the fusion processing of multi-sensor measurement information, and the filtering principle is shown in Figure 7. In the velocity measurement and location method based on federated Kalman filter fusion, a separate local linear Kalman filter state estimation is carried out for each sensor measurement first, and the local optimal velocity measurement and location estimation of each subfilter is outputted, then, the local optimal velocimetry location estimation $\mathrm{A} \underset{i}{\hat{X}}$ of each sub-filter and its covariance matrix $P_{i}$ are sent to the main filter together, according to certain rules of information fusion processing, and finally get the global optimal velocity measurement location estimation $\underset{g}{X}$ and the corresponding covariance matrix $P_{g}$. The idling/taxiing phenomenon was detected by the wheel shaft speed sensor and accelerometer, and the detected idling/taxiing phenomenon was processed by the doppler radar speed sensor. The wheel diameter is corrected and adjusted by querying the position information of the transponder and the global final position estimation. Finally, the information allocation principle is used to return the allocation information for each local filter. In 


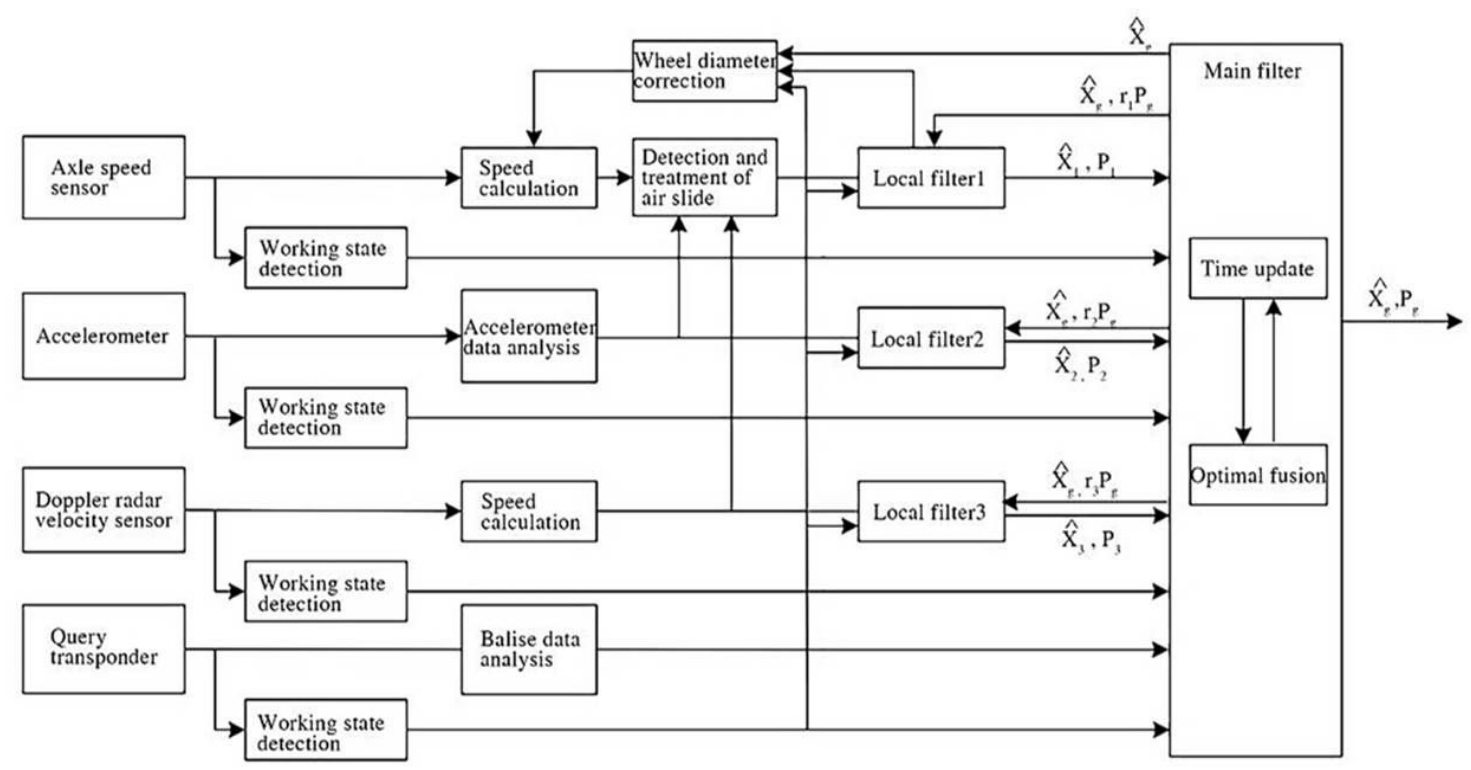

Figure 7: Principle of filtering

this way, the advantages of each sensor can be fully utilized to complement each other so as to achieve the purpose of improving the accuracy of velocity measurement and positioning [28].

\section{Simulation analysis and results}

\subsection{Simulation module design}

According to the train motion model and sensor measurement model, the simulation model of the system is established in the matlab/simulink environment, as shown in Figure 8. The model consists of three parts: data input module, filter processing module and information fusion module. Wherein, the ideal acceleration curve is generated in the computer and the position and speed of the train are calculated. Combined with the noise of the system simulation, the average difference of acceleration noise is $0.1 \mathrm{~m} / \mathrm{s}^{2}$, the standard deviation of speed noise is $0.1 \mathrm{~m} / \mathrm{s}$, and the standard deviation of position noise is $0.5 \mathrm{~m}$. The data of each sensor's measurement sequence are fed into three local filters for filtering processing. The filtering results of the local filter are fed into the main filter module for information fusion to obtain the global optimal estimation, and the data files are stored in the $\mathrm{XG}$ and $\mathrm{PG}$ modules. The function of the zero-order hold signal in the simulation process is to sample the data of the input filter, so that all the data have the same update period [29].

\subsection{Result analysis and discussion}

In order to verify the filtering algorithm more effectively, simulation experiments with and without information fusion were carried out respectively. The running time of the train was $1000 \mathrm{~s}$, the simulation step size of the model was $0.005 \mathrm{~s}$, the output period of the wheel shaft speed sensor was $0.005 \mathrm{~s}$, and the output period of the accelerometer was $0.01 \mathrm{~s}$. The output cycle of doppler radar is $0.1 \mathrm{~s}$, the output cycle of the query transponder is 1 s, and the fusion cycle of the main filter is 1s. The system simulation results are shown in Figures 9 and 10.

From Figures 9 and 10 it can be inferred that speed positioning method based on fusion filtering can effectively reduce the influence of system error and measurement error, and the precision of state estimation than no filtering velocity under the condition of positioning accuracy is high, the location, velocity estimation accuracy is improved, shows that the method can restrain the error caused by noise accumulation.

Also, the simulation of real time operation of data generated by the trained is done for the complete simulation time of 1000 secs and in between a period of fusion is observed for $1 \mathrm{sec}$. This simulated experimentation is observed for speed curve and the position curve depicted in Figures 11 and 12, respectively.

In the graphical representation of Figures 11 and 12, the original set waveform is depicted by blue-colored line and the orange-colored dotted line represents the fusion undergone output waveform. From the waveforms, it is ob- 


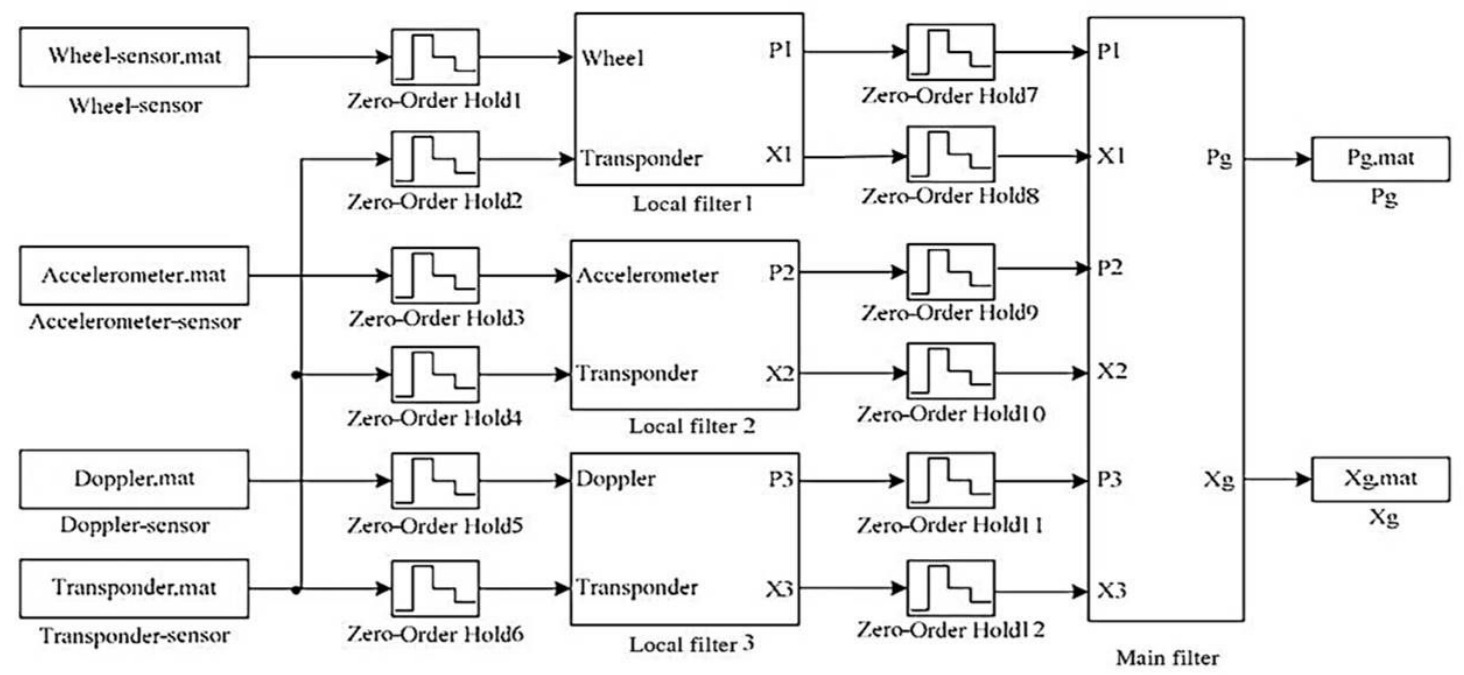

Figure 8: Simulation model

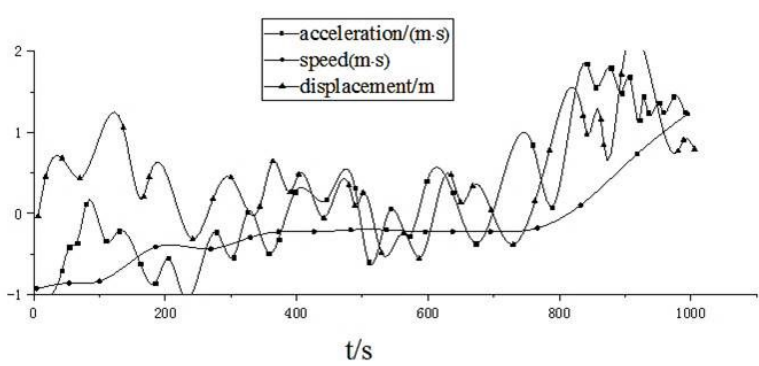

Figure 9: Simulation results of the system without filtering

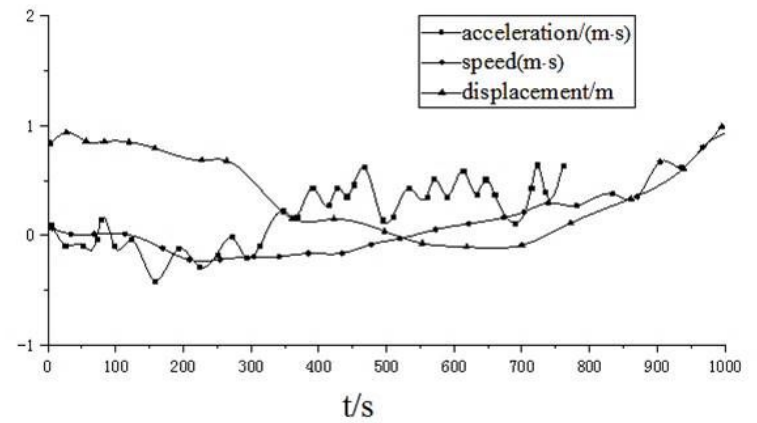

Figure 10: Simulation results of the system under filtering

served that the higher degree of fitting is observed and the proposed combination of position and speed measurement has achieved good levels of accuracy while maintaining a stable corrected waveform even under noise interference influence. A robust and autonomous operating ability is maintained by the proposed methodology while providing higher rate of accuracy.

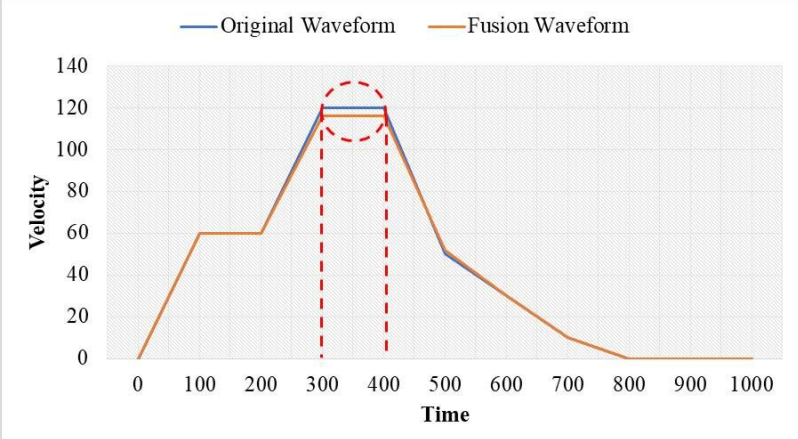

Figure 11: Simulated outcomes for speed curve

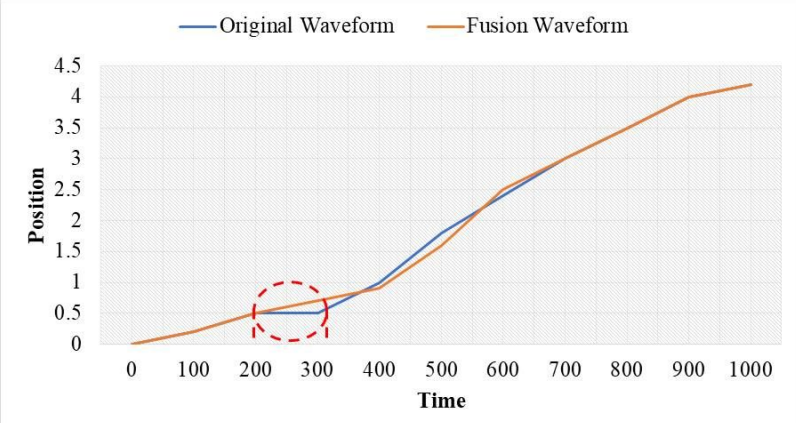

Figure 12: Simulated outcomes for position curve

\section{Conclusions}

This article presents the design and implementation of an embedded multi-sensor information based on the fusion study of the train speed and positioning. The proposed system is based on the embedded multiple sensor 
information fusion of train speed positioning system, through multiple sensor technology in the process of train running wear, idler wheel diameter and sliding phenomena, and uses the federal Kalman filtering fusion algorithm for information processing. The experimental simulation results show that this method can detect the idling/taxiing phenomenon of the train timely and effectively, restrain the error accumulation caused by noise, and improve the accuracy of the train speed measurement and ranging. However, the simplified processing of the train motion model in this method has a certain impact on the system simulation, but a robust and autonomous operating ability is maintained while providing higher rate of accuracy. The future prospect of this work will be focused on accurate identification of the train model and the optimization of the filter fusion algorithm.

Funding information: The authors state no funding involved.

Author contributions: All authors have accepted responsibility for the entire content of this manuscript and approved its submission.

Conflict of interest: The authors state no conflict of interest.

\section{References}

[1] Sun Y, Cui Y. Evaluating the coordinated development of economic, social and environmental benefits of urban public transportation infrastructure: case study of four Chinese autonomous municipalities. Transp Policy. 2018;66:116-26.

[2] Hu X, Chen T, Zhao N. Simulation Analysis of Train Speed Measurement Technology Based on Urban Rail Transit System. Int Conf Intell Trans, Big Data \& Smart City (ICITBS) 2016;2528.

[3] Poongodi M, Sharma A, Hamdi M, Maode M, Chilamkurti N. Smart healthcare in smart cities: wireless patient monitoring system using loT. J Supercomput. 2021;1-26.

[4] Wang D, Liu S. Study on Application of Automatic Turn-back Technology in Intercity Railways. Railway Com Signal Eng Tech. 2018;15(12).

[5] Sun H, Fan M, Sharma A. Design and implementation of construction prediction and management platform based on building information modelling and three-dimensional simulation technology in industry 4.0. IET Collab Intell Manuf. 2021;1-9

[6] Ren X, Li C, Ma X, Chen F, Wang H, Sharma A, et al. Design of multi-information fusion based intelligent electrical fire detection system for green buildings. Sustainability (Basel). 2021;13(6):3405.

[7] Orosz T, Rassõlkin A, Kallaste A, Arsénio P, Pánek D, Kaska J, et al. Robust Design Optimization and Emerging Technologies for Electrical Machines: Challenges and Open Problems. Appl Sci (Basel). 2020;10(19):6653.

[8] Huang C, Huang Y. Research and design of data communication subsystem of urban rail transit CBTC system. Int J Sys Assur Eng Manag. 2021;1-11.

[9] Xu X, Li L, Sharma A. Controlling messy errors in virtual reconstruction of random sports image capture points for complex systems. Int J Sys Assur Eng Manag. 2021;1-8.

[10] Xue QL, Wang, PL. The Research and Realization of Track Layout Editor TOPOLOGY in Urban Rail Transit Signaling System. J Railway Locomotives. 2011;5:44-46.

[11] Ding S, Wang W, Yang X, Wang S, Chen L. Study on strength and durability of fair-faced concrete in underground station of urban rail transit. IOP Conf Series Mater Sci Eng. 2020;794:012068.

[12] Lin Y, Wang D, Zhang W. Research on speed and distance measurement algorithm based on multi-sensor information fusion. Int Conf Ener, Env Sus Dev (ICEESD 2017). 2017;265-273. Atlantis Press. https://doi.org/10.2991/iceesd-17.2017.51.

[13] Xiong Z, Zheng J, Song D, Zhong S, Huang Q. Passenger flow prediction of urban rail transit based on deep learning methods. Smart Cities. 2019;2(3):371-87.

[14] Dai C, Dou F, Song X, Long Z. Analysis and design of a speed and position system for maglev vehicles. Sensors (Basel). 2012;12(7):8526-43.

[15] Liu C, Cheng S, Li K. Position and Speed Measuring Method of Maglev Train Based on Federal Kalman Filter and Information Fusion. IOP Publishing. J Phys Conf Ser. 2020;1621(1):012067.

[16] Harrer F, Pfeiffer F, Löffler A, Gisder T, Biebl E. Automotive synthetic aperture radar system based on $24 \mathrm{GHz}$ series sensors. Advanced Microsystems for Automotive Applications 2017. Cham: Springer; 2018;23-36.

[17] Yang Y, Yan J, Guo J, Kuang Y, Yin M, Wang S, et al. Driving behavior analysis of city buses based on real-time GNSS traces and road information. Sensors (Basel). 2021;21(3):687.

[18] Liu B, Wei S, Su G, Wang J, Lu J. An improved fast selfcalibration method for hybrid inertial navigation system under stationary condition. Sensors (Basel). 2018;18(5):1303.

[19] Zhang Y, Wang B, Zhang L. High-precision GPS measurement method without geographical restrictions using crowd-sensing technology. Earth Sci Res J. 2020;24(4):491-7.

[20] Lyshevski SE, Hughes DH, Malowicki J, Bedi V, Nikulin VV. Analysis of data-driven processing in inertial measurement systems for wireless optical communication applications. Micro-and Nanotechnology Sensors, Systems, and Applications XI, 109823G. Int Soc Opt Photonics. 2019;10982.

[21] Chen L, Thombre S, Järvinen K, Lohan ES, Alén-Savikko A, Leppäkoski H, et al. Robustness, security and privacy in location-based services for future loT: A survey. IEEE Access. 2017;5:8956-77.

[22] Wang Y, Wang P, Li Z, Chen Z, He Q. Forecasting Urban Rail Transit Vehicle Interior Noise and Its Applications in Railway Alignment Design. J Adv Transp. 2020.

[23] Zhang G, Guo L, Liu Y, Gao X, Wang Z, Wang Z, et al. Research of speed measurement and prediction system for railway train based on single chip microcomputer [IOP Publishing.]. J Phys Conf Ser. 2020;1650(3):032120.

[24] Ma S, Wang X, Wang X, Liu H, Zhang R. A Framework for Diagnosing Urban Rail Train Turn-Back Faults Based on Rules and 
Algorithms. Appl Sci (Basel). 2021;11(8):3347.

[25] Muniandi G, Deenadayalan E. Train distance and speed estimation using multi sensor data fusion. IET Radar Sonar \& Navigation. 2019;13(4):664-71.

[26] Wang B, Yang G, Zhou J, Ye M, Cheng H. Congested Situation Identification of Urban Rail Transit Carriage Based on Deep Learning. COTA Intl Conf Trans Prof. 2020;2851-2862.

[27] Zhang Y. Research on Statistical Index and Evaluation System of Urban Rail Transit Train Delay Based on Operation Diagram. Int Conf Intell Trans Eng (ICITE). 2019.

[28] Ning LI. Study on connectivity reliability of urban rail transit network based on complex network theory. Construction \& Design for Engineering. 2019.

[29] Wei Q, Pan Z, Liu H, Ming LI, Wei R. Study on the classification method of urban rail transit based on technical principle. Urban Mass Transit. 2019. 